\title{
SVN sordenend \\ Effects and safety of aspirin use in patients after cerebrovascular bypass procedures
}

Junlin Lu (D) , ${ }^{1}$ Guangchao Shi, ${ }^{2}$ Yuanli Zhao, ${ }^{1,2,3,4,5,6}$ Rong Wang, ${ }^{1,2,3,4,5}$
Dong Zhang, ${ }^{1,3,4,5}$ Xiaolin Chen, ${ }^{1,3,4,5}$ Hao Wang, ${ }^{1,3,4,5,6}$ Ji Zong Zhao ${ }^{1,3,4,5,6}$

To cite: Lu J, Shi G, Zhao Y, et al. Effects and safety of aspirin use in patients after cerebrovascular bypass procedures. Stroke \& Vascular Neurology 2021;0. doi:10.1136/svn-2020-000770

$\mathrm{JL}$ and GS contributed equally.

Received 28 November 2020

Revised 7 April 2021

Accepted 20 April 2021

Check for updates

(C) Author(s) (or their employer(s)) 2021. Re-use permitted under CC BY-NC. No commercial re-use. See rights and permissions. Published by BMJ.

${ }^{1}$ Neurosurgery, Beijing Tiantan Hospital, Capital Medical University, Beijing, China ${ }^{2}$ Neurosurgery, Peking University International Hospital, Beijing,

China

${ }^{3}$ China National Clinical

Research Center for

Neurological Diseases, Beijing,

China

${ }^{4}$ Stroke Center, Beijing Institute for Brain Disorders, Beijing,

China

${ }^{5}$ Beijing Key Laboratory of Translational Medicine for Cerebrovascular Disease, Beijing, China

${ }^{6}$ Beijing Translational

Engineering Enter for 3D Printer in Clinical Neuroscience, Beijing, China

Correspondence to

Dr Hao Wang;

cmu990103@163.com

\section{ABSTRACT}

Object Superficial temporal artery to middle cerebral artery (STA-MCA) bypass is the most effective treatment for Moyamoya disease (MMD). In this study, we aimed to assess whether aspirin improves STA-MCA bypass patency and is safe in patients with MMD.

Methods We performed a retrospective medical record review of patients with ischaemic-onset MMD who had undergone STA-MCA bypass at two hospitals between January 2011 and August 2018, to clarify the effects and safety of aspirin following STA-MCA bypass. The neurological status at the last follow-up (FU) was compared between patients with FU bypass patency and occlusion. Results

Among 217 identified patients (238 hemispheres), the mean age was $41.4 \pm 10.2$ years, and $51.8 \%$ were male; the indications for STA-MCA bypass were stroke (48.2\%), followed by a transient ischaemic attack (44.0\%). Immediate bypass patency was confirmed in all cases. During the FU period $(1.5 \pm 1.5 \mathrm{y}), 15$ cases were occluded at FU imaging, resulting in an overall cumulative patency rate of $94 \%$. The patency rates were $93 \%$ and $94 \%$ in the short-term FU group ( $n=131$, mean FU time $0.5 \pm 0.2$ years) and long-term FU group ( $n=107$, mean FU time 4.1 \pm 3.5 years), respectively. The STA-MCA bypass patency rate in the aspirin group was higher than that in the non-aspirin group (98.7\% vs $89.7 \%$; HR 1.57 ; $95 \% \mathrm{Cl} 1.106$ to 2.235 ; $\mathrm{p}=0.012$ ). No significant difference in the FU haemorrhagic events was observed between the aspirin and non-aspirin groups.

Conclusions Among adult patients with ischaemic-onset MMD undergoing STA-MCA bypass procedures, aspirin might increase the bypass patency rate, without increasing the bleeding risk. FU bypass patency may be associated with a better outcome. Additional studies, especially carefully designed prospective studies, are needed to address the role of aspirin after bypass procedures.

\section{INTRODUCTION}

Revascularisation can provide significant benefits in survival and quality of life and is favoured over conservative medical therapy in patients with Moyamoya disease (MMD). ${ }^{12}$ Superficial temporal artery to middle cerebral artery (STA-MCA) bypass has been established as the standard of care in MMD. The patency of the bypass is a key factor to determine the success of the intervention, as well as to assess the long-term survival in patients. ${ }^{3}$ Although the safety and feasibility of bypass procedures have improved owing to advances in surgical techniques and neuroanaesthesia, large patient cohort analyses on cerebral bypass patency with long-term follow-up (FU) results remain limited. A study published by the extracranial-intracranial (EC/IC) Bypass Study Group in 1985 reported a postoperative bypass patency rate of $96 \%$ among the 652 patients who underwent STA-MCA bypass, with the longest FU of 7.5 years. ${ }^{4}$ More recently, in a study conducted at the Barrow Neurological Institute, the authors observed that the overall cumulative patency rate of 97\% among 430 patients, surgical indication, low-flow bypass and the number of anastomoses were reportedly associated with higher patency rates. ${ }^{3}$

Notably, antiplatelet therapy with aspirin improves vein graft patency, and current guidelines recommend continuing aspirin after coronary artery bypass grafting. ${ }^{5}$ Despite several institutions reporting their experience on the use of aspirin after revascularisation surgery in neurosurgical patients, ${ }^{67}$ the effect of antiplatelet aspirin therapy in the setting of neurosurgical patients after revascularisation has not been confirmed. We have accumulated a large amount of experience with cerebral revascularisation and aimed to provide some information on the use of aspirin in neurosurgical patients after revascularisation. Thus, this study analysed the long-term patency of STA-MCA bypass procedures performed in patients with MMD over 10 years and assessed the effect of aspirin on bypass patency.

\section{MATERIALS AND METHODS}

\section{Patients and materials}

We conducted a retrospective study with longterm FU to assess the effect of aspirin on the bypass patency of MMD. Herein, the participants included were from a multicentre cohort of consecutive patients with MMD, 


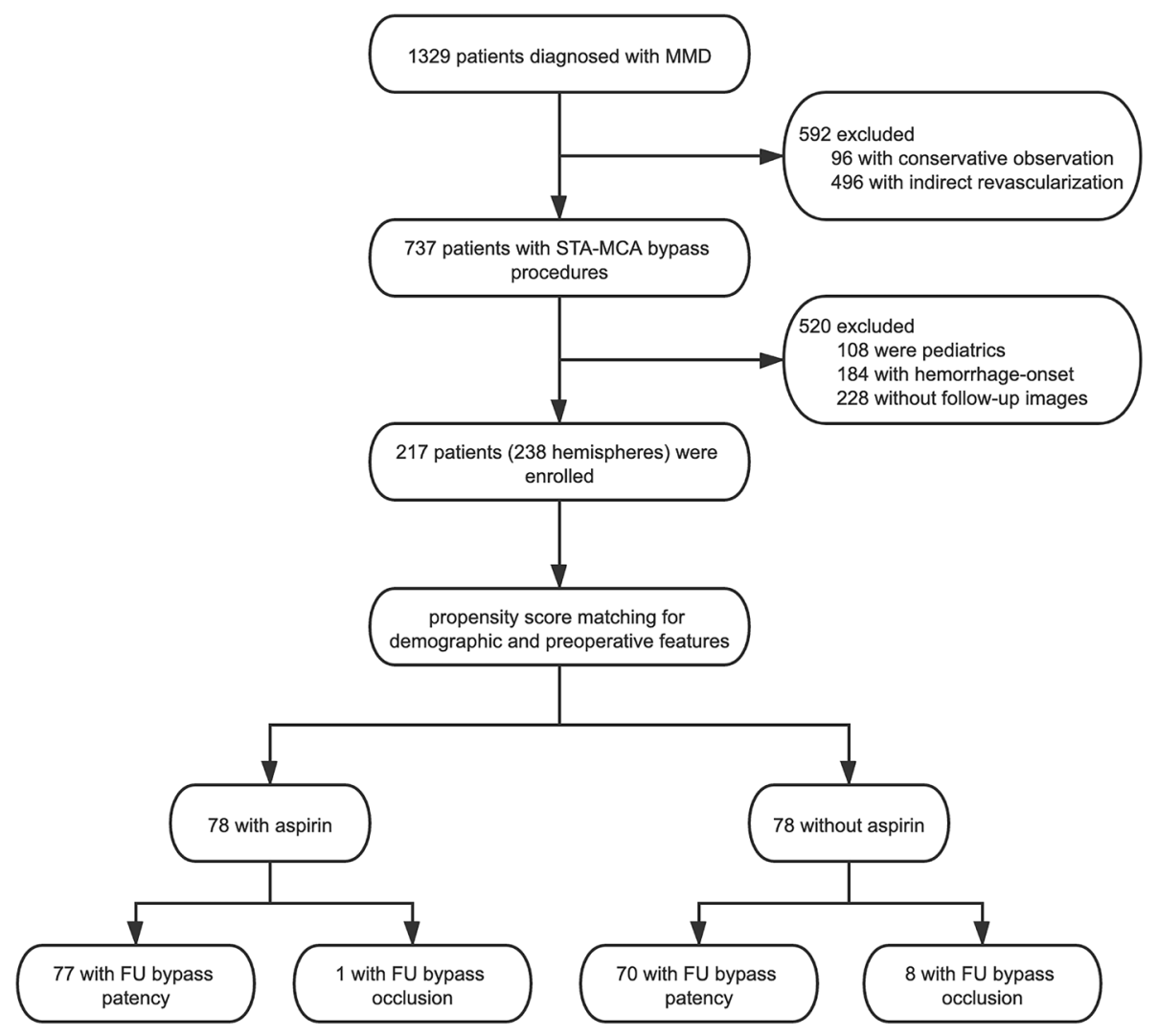

Figure 1 Flow diagram of the study population. FU, follow-up; MMD, Moyamoya disease; STA-MCA, superficial temporal artery to middle cerebral artery.

treated between 2011 and 2018 (figure 1). The goal was to provide evidence for the use of aspirin for the postoperative management of MMD. The inclusion criteria were as follows: (1) patients with a diagnosis of MMD by digital subtraction angiography (DSA)/MR angiography (MRA)/CT angiography (CTA) according to the guidelines for MMD (criteria of the Research Committee on Spontaneous Occlusion of the Circle of Willis, 2012) ${ }^{8}$; (2) patients who received only STA-MCA bypass procedures. The exclusion criteria were as follows: (1) patients without FU DSA/CTA/MRA; (2) paediatric patients or patients who previously presented cerebral haemorrhage symptoms.

Baseline clinical characteristics and imaging data were reviewed, including age, sex, onset manifestation, medical history, neurological status and imaging findings. The onset manifestations were divided as follows: transient ischaemic attack, stroke, others (headache or epilepsy) and asymptomatic. Suzuki stage was recorded as previously described. ${ }^{9}$

\section{Surgical procedures}

Our principles for surgical strategies were reported in previous studies. ${ }^{10} 11$ Briefly, revascularisation surgery was performed on the hemisphere with symptoms and haemodynamic hypoperfusion. All bypass procedures were performed under general anaesthesia with intravenous propofol and remifentanil. Patients were maintained under conditions of normovolaemia and normocapnia, with intraoperative systolic blood pressure maintained between 120 and $140 \mathrm{~mm} \mathrm{Hg}$. Bypass patency was confirmed by employing intraoperative DSA or indocyanine green angiography. For patients without obvious intracranial haemorrhage following a CT scan performed 4 hours after surgery, aspirin was started postoperatively on the day of surgery (100 mg once a day).

\section{FU Methods}

We performed a manual chart review to collect data on imaging studies, through either DSA, CTA or MRA, completed preoperatively, postoperatively, and at FU. The FU bypass patency was determined by two independent radiology-trained neurosurgeons. Aspirin was started postoperatively on the day of surgery, indefinite administration was suggested. However, not all patients followed the physician's advice, and some patients stopped administering aspirin after discharge. Data on whether these patients continuously administered aspirin after the bypass procedures were obtained from records at discharge and the questionnaire during FU. Patients were divided into two groups according to whether they continuously administered postoperative aspirin. Patients in the aspirin group consistently administered the medication (100 mg once a day) from discharge to their last FU. In 
contrast, patients in the non-aspirin group did not insist on taking the medication and quit the aspirin administration on their own within 1 month after discharge.

All patients were solicited for annual $\mathrm{FU}$ visits in the outpatient clinic. For patients who were unavailable for in-person interviews, we attempted to obtain basic clinical information over the phone to assess the onset of ischaemic events, haemorrhagic events and survival status. Clinical functional outcomes after revascularisation were recorded using the modified Rankin scale (mRS) score. Patients' outcome was divided into two categories, good (mRS 0-2) and poor (mRS 3-6).

\section{Data analysis}

All analyses were conducted using IBM SPSS statistical software V.26.0 (IBM). Statistical significance was set at $\mathrm{p}<0.05$, for $95 \%$ CI. The original baseline differences between the aspirin and non-aspirin groups were evaluated using a t-test for continuous variables and $\chi^{2}$ test for categorical variables.

Propensity score matching was employed to reduce imbalances in baseline characteristics between patients with and without continuous aspirin administration. The propensity scores for the continuous use of aspirin after STA-MCA bypass were estimated using a logistic multivariate regression model containing demographic characteristics (age and sex), preoperative angiopathy (Suzuki stage and posterior circulation involvement) and medical history (smoking and diabetes). Using the nearestneighbour method without replacement for propensity score matching, pairs of patients were matched with a match tolerance of 0.02 and a ratio of $1: 1{ }^{12}$

In the matched pairs, the exact McNemar test and paired t-test were used to compare baseline characteristics. Factors associated with FU bypass patency were examined using univariate and multivariate Cox regression analyses. The cumulative incidence rate of outcome events was presented using the Kaplan-Meier curves. Logrank test statistics were used to determine whether the Kaplan-Meier transition curves differed between the two groups.

To further determine the relationship between $\mathrm{FU}$ bypass patency and clinical outcomes, FU ischaemic events, haemorrhagic events and mRS scores were analysed using a logistic regression model that was adjusted for potential factors related to the FU neurological function.

\section{RESULTS}

\section{Patient baseline and propensity score-matched cohort}

In total, 217 patients with MMD underwent 238 STAMCA bypass procedures. Of these, 128 patients started antiplatelet therapy with aspirin postoperatively, which was then continued indefinitely. Baseline characteristics differed with respect to age, cigarette smoking status, Suzuki stage, posterior involvement and FU bypass patency between patients who continued administering aspirin (aspirin group; $100 \mathrm{mg}$ once a day) and those who did not continue administering aspirin (no aspirin group) (table 1). Using propensity score matching, 78 procedures performed with continuous postoperative aspirin administration were matched to procedures that did not administer aspirin therapy. No significant differences were observed between the propensity scorematched groups in terms of patient age, sex, preoperative symptoms, preoperative mRS score, medical history, Suzuki stage or posterior involvement.

\section{FU STA-MCA bypass patency}

The two observers were in agreement regarding the $\mathrm{FU}$ bypass patency $(\kappa=0.715 ; \mathrm{p}<0.001)$. Of the 238 STA-MCA bypass procedures in the series, no cases of bypass occlusion were recorded in the immediate postoperative period according to intraoperative DSA or indocyanine green angiography. Fifteen cases that underwent bypass were occluded on FU imaging, resulting in an overall cumulative patency rate of $94 \%$. We categorised the patients into two groups based on the FU time point: 131 shortterm ( $>3$ months, $<1$ year) FU patients and 107 long-term ( $>1$ year) FU patients. Nine bypass cases found to be were occluded at short-term FU (average, $0.5 \pm 0.2$ years), which resulted in a $93 \%$ patency rate in this cohort. Among 101 of the 107 cases, long-term patency could be assessed with an average $\mathrm{FU}$ time of 2.8 years \pm 1.8 years (range $1-9$ years).

\section{Aspirin improves the FU STA-MCA bypass patency rate}

No significant difference was observed in the bypass patency rate between the aspirin and non-aspirin groups in the short-term FU $(p=0.266)$. Aspirin administration improved the bypass patency rate during long-term FU $(p=0.045)$. Furthermore, we observed a statistically significant difference in the cumulative patency rates based on aspirin administration in the propensity score-matched groups (table 1). There was a higher rate of bypass patency in the aspirin group $(77 / 78,98.7 \%)$ than in the non-aspirin group $(70 / 78,89.7 \%)(\mathrm{p}=0.016)$.

We created an adjusted multiple Cox regression model by utilising bypass patency as the primary outcome to account for aspirin administration in our cohort (table 2). The adjusted model confirmed aspirin administration as an independent predictor of bypass patency (HR 1.57; 95\% CI 1.106 to 2.235; $\mathrm{p}=0.012$ ). Based on our analysis, age, sex, cigarette smoking status, diabetes, hypertension, hyperlipidaemia, Suzuki stage and posterior involvement were not associated with increased potential of the bypass patency rate $(\mathrm{p}>0.05)$.

\section{Aspirin and FU STA-MCA bypass patency versus clinical outcome}

During the average $2.7 \pm 1.3$ years $\mathrm{FU}$ period, 26 patients $(26 / 217,12.0 \%)$ were lost to FU. No significant difference in the incidence of ischaemic and haemorrhagic events was observed between the aspirin and non-aspirin groups in the entire cohort (figure 2A,B). Table 3 summarises the clinical outcomes of patients who underwent 
Table 1 Baseline characteristics

\begin{tabular}{|c|c|c|c|c|c|c|}
\hline \multirow[b]{3}{*}{ Characteristic } & \multicolumn{3}{|c|}{ Before propensity score matching } & \multicolumn{3}{|c|}{ After propensity score matching } \\
\hline & \multirow{2}{*}{$\begin{array}{l}\text { Aspirin } \\
\text { Group }\end{array}$} & \multirow{2}{*}{$\begin{array}{l}\text { Non-aspirin } \\
\text { Group }\end{array}$} & \multirow[b]{2}{*}{ P value } & \multirow{2}{*}{$\begin{array}{l}\text { Aspirin } \\
\text { Group } \\
\end{array}$} & \multirow{2}{*}{$\begin{array}{l}\text { Non-aspirin } \\
\text { Group } \\
\end{array}$} & \multirow[b]{2}{*}{$P$ value } \\
\hline & & & & & & \\
\hline No of ops & 141 & 97 & & 78 & 78 & \\
\hline No of pts & 128 & 89 & & 78 & 78 & \\
\hline Mean age, years & $41.4 \pm 10.2$ & $36.5 \pm 8.7$ & $<0.001$ & $37.5 \pm 9.6$ & $37.6 \pm 8.9$ & 0.996 \\
\hline Sex & & & 0.149 & & & 0.631 \\
\hline Male & $73(51.8)$ & $41(42.3)$ & & $39(50)$ & $36(46.2)$ & \\
\hline Female & $68(48.2)$ & $56(57.7)$ & & $39(50)$ & $42(53.8)$ & \\
\hline Preop symptoms & & & 0.052 & & & 0.051 \\
\hline TIA & $62(44.0)$ & $34(35.1)$ & & $37(47.4)$ & $26(33.3)$ & \\
\hline Stroke & $68(48.2)$ & $50(51.5)$ & & $37(47.4)$ & $41(52.6)$ & \\
\hline Others & $6(4.3)$ & $12(12.4)$ & & $2(2.6)$ & $10(12.8)$ & \\
\hline Asymptomatic & $5(3.5)$ & $1(1.9)$ & & $2(2.6)$ & $1(1.3)$ & \\
\hline Preop mRS score & & & 0.417 & & & 0.186 \\
\hline 0 & $8(5.7)$ & $3(3.1)$ & & $4(5.1)$ & $2(2.6)$ & \\
\hline $1-2$ & $127(90.1)$ & $87(89.7)$ & & 73 (93.6) & $71(91.0)$ & \\
\hline 3 & $6(4.3)$ & $7(7.2)$ & & $1(1.3)$ & $5(6.4)$ & \\
\hline \multicolumn{7}{|l|}{ Medical history } \\
\hline Smoking & $28(19.9)$ & $10(10.3)$ & 0.048 & $11(14.1)$ & $10(12.8)$ & 0.815 \\
\hline Diabetes & $19(13.5)$ & $6(6.2)$ & 0.071 & $6(7.7)$ & $5(6.4)$ & 0.754 \\
\hline Hypertension & 47 (33.3) & $31(32.0)$ & 0.824 & $22(28.2)$ & 27 (34.6) & 0.388 \\
\hline Hyperlipidaemia & $13(9.2)$ & $5(5.2)$ & 0.244 & $6(7.7)$ & $4(5.1)$ & 0.513 \\
\hline Suzuki stage & & & 0.042 & & & 0.418 \\
\hline 1 & $1(0.7)$ & $2(2.1)$ & & $1(1.3)$ & $2(2.6)$ & \\
\hline 2 & $9(6.7)$ & $6(6.3)$ & & $5(6.4)$ & $5(6.4)$ & \\
\hline 3 & $38(28.1)$ & $29(30.2)$ & & $24(30.8)$ & $23(29.5)$ & \\
\hline 4 & $42(31.1)$ & $36(37.5)$ & & $26(33.3)$ & $25(32.1)$ & \\
\hline 5 & $31(23.0)$ & $23(24.0)$ & & $18(23.1)$ & $23(29.5)$ & \\
\hline 6 & $14(10.4)$ & $0(0)$ & & $4(5.1)$ & $0(0)$ & \\
\hline Posterior involvement & $20(14.8)$ & $28(29.2)$ & 0.008 & $16(20.5)$ & $14(17.9)$ & 0.685 \\
\hline Follow-up bypass patency & $136(96.5)$ & $87(89.7)$ & 0.035 & $77(98.7)$ & $70(89.7)$ & 0.016 \\
\hline
\end{tabular}

Data are presented as $\mathrm{n}(\%)$ unless otherwise indicated. The mean values are presented as SDs. mRS, modified Rankin scale; Ops, operations; Pts, patients; TIA, transient ischaemia attack.

patency and occlusion bypasses. Patients in the occlusion bypass group presented a higher incidence of haemorrhagic events $(6.7 \%$ vs $0.5 \%)$ and poor prognosis $(6.7 \%$ vs $0.5 \%)$. However, no significant differences were observed after adjusting for the potential factors.

\section{DISCUSSION}

In this retrospective multicentre study, we investigated the potential benefits of aspirin in preventing graft occlusion after STA-MCA bypass. This study demonstrated the positive effect of aspirin on the rate of graft patency, as well as on the composite of graft patency and clinical events.

As previously mentioned, STA-MCA bypass is considered the most effective treatment strategy for MMD, significantly reducing the risk of future stroke and the frequency of transient ischaemic attacks when compared with conservative medical therapy alone. However, ischaemic stroke reportedly presents an occurrence risk of $2 \%-5 \%$ during the $\mathrm{FU}$ period. ${ }^{13}{ }^{14}$ Bypass patency may one potential factor affecting the occurrence of ischaemic stroke during the FU period. As an antiplatelet drug, aspirin is a powerful inhibitor of inflammation and has been administered as standard antiplatelet therapy after coronary bypass surgery to increase the success rate of the bypass graft. ${ }^{5}$ Furthermore, aspirin has been indicated in the conservative medical management of MMD as a treatment option for a prolonged period, as it might improve microcirculation and prevent embolism, leading 
Table 2 Cox regression analysis for factors increasing the rate of bypass patency in the propensity score-matched cases

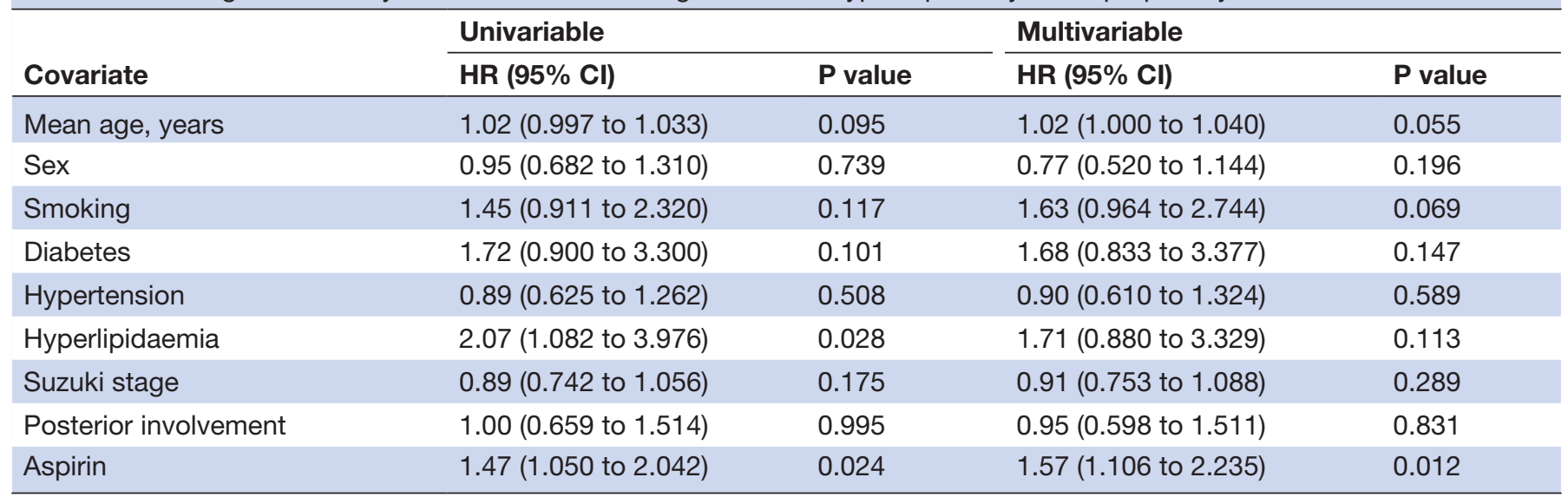

to better outcomes in patients with MMD. ${ }^{15}{ }^{16}$ However, it remains controversial whether aspirin administration is appropriate in patients after STA-MCA bypass procedures as antiplatelet therapy presents the risk of a subsequent cerebral haemorrhage. ${ }^{615}$ We found a limited number of studies investigating the role of antiplatelet agents such as aspirin after bypass procedures in neurosurgical patients. Results from our previous study investigating the effect of aspirin after STA-MCA bypass revealed an overall patency rate of $96.9 \%$ and no significant difference between the aspirin and non-aspirin groups. ${ }^{17}$ However, it should be noted that less than $50 \%$ of patients underwent postoperative angiography in our previous study. Furthermore, the small sample size (98 cases in total) hindered the detection of statistically significant differences in the patency rate between aspirin and non-aspirin groups. Thus, we conducted a multicentre study to increase the sample size. The overall bypass patency rate was $94 \%$ in our multicentre cohort during an average FU period of 1.5 years. Our results showed that aspirin administration can improve the FU STA-MCA bypass patency rate, especially in the long-term FU period. Aspirin can irreversibly inhibit platelet cyclooxygenase, prevent platelet function, and demonstrates an antithrombotic effect. In the present study, the immediate bypass patency rate was $100 \%$, which might be attributed to all the patients receiving postoperative aspirin therapy during hospitalisation. After discharge, some patients failed to continue

A
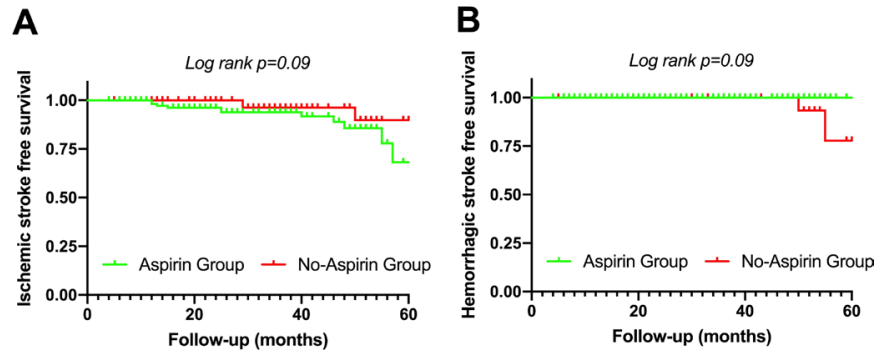

Figure 2 The Kaplan-Meier curve showing the cumulative rate of ischaemic events $(A)$ and haemorrhagic events $(B)$ between aspirin and non-aspirin groups in the entire cohort. to aspirin administration, leading to a difference in the FU STA-MCA bypass patency rate between the two groups.

Currently, a variety of mechanisms could lead to bypass occlusion, including thrombosis, intimal thickening and atherosclerosis. Harvesting of the bypass graft, as well as ischaemia reperfusion, can damage the vascular endothelial cells (VECs) of the bypass graft, resulting in a decreased anticoagulant ability. Furthermore, the dysfunction of VECs impedes the expression of endotheliumderived active factors and the release of inflammatory mediators, which in turn stimulate the proliferation and migration of smooth muscle cells, resulting in intimal thickening. Thus, VECs play an essential role in bypass patency. Previous experiments have established that lowdose aspirin can promote VEC proliferation, migration and adhesion. ${ }^{18}$ In contrast, high-dose aspirin reportedly decreases VEC proliferation, migration, and adhesion. ${ }^{19}$ However, it should be noted that the dose and time of aspirin administration following bypass procedures has not been standardised, and most regimens for postoperative antiplatelet therapy remain empirical. Some experts have suggested initiating postoperative aspirin therapy (325 mg once a day) for life after bypass procedures, ${ }^{6}$ while low-dose aspirin (100 mg once a day) is commonly used in Asian countries. ${ }^{710}$ A prospective randomised clinical trial is needed to further investigate the effect of different aspirin doses on bypass patency.

Consistent with our previous study, ${ }^{17}$ the present study showed that postoperative aspirin therapy (100 mg once a day) does not increase the risk of haemorrhage; however, it does not appear to reduce postoperative ischaemic events. Considering that the occurrence rate of postoperative ischaemic events in patients with MMD is less than $5 \%$, we speculate that the relatively small size of the present study fails to a detect a significant difference. We further investigated the STA-MCA bypass patency in association with clinical outcomes. The statistics analysis barely detected any significance in the occurrence rate of ischaemic and haemorrhagic events between the two groups, which was probably attributed to the unequal sample size and low ischaemia and bleeding rates. In MMD, 
Table 3 Follow-up outcomes of patients with patency and occlusion bypass in the entire cohort

\begin{tabular}{|c|c|c|c|c|c|c|}
\hline & Total & Patency group & Occlusion group & \multirow[b]{2}{*}{$P$ value } & \multirow[b]{2}{*}{ Adjusted OR (95\% CI) } & \multirow{2}{*}{$\begin{array}{l}\text { Adjusted } \\
\mathbf{P} \text { value }\end{array}$} \\
\hline & $(n=191)$ & $(n=176)$ & $(n=15)$ & & & \\
\hline Ischaemic events & $13(6.8)$ & $12(6.8)$ & $1(6.7)$ & 0.982 & $0.75(0.085 \text { to } 6.582)^{\star}$ & 0.794 \\
\hline Haemorrhagic events & $2(1.0)$ & $1(0.6)$ & $1(6.7)$ & 0.026 & $0.15(0.008 \text { to } 2.882)^{\star}$ & 0.209 \\
\hline mRS score at last follow-up & & & & 0.026 & $0.03(0.001$ to 1.373$) \dagger$ & 0.073 \\
\hline $0-2$ & $189(99.0)$ & $175(99.4)$ & 14 (93.3) & & & \\
\hline $3-6$ & $2(1.0)$ & $1(0.6)$ & $1(6.7)$ & & & \\
\hline Disability-free recovery & $171(89.5)$ & $157(89.2)$ & $14(93.3)$ & 0.616 & 0.57 (0.069 to 4.688$) \dagger$ & 0.599 \\
\hline Improved neurological function & $137(71.7)$ & $128(72.7)$ & $9(60.0)$ & 0.293 & $1.81(0.603$ to 5.416$) \dagger$ & 0.291 \\
\hline
\end{tabular}

Data are presented as $\mathrm{n}(\%)$ unless otherwise indicated. The mean values are presented as SDs.

*Adjusted for aspirin administration, hypertension and posterior circulation involvement.

†Adjusted for age, sex and preoperative mRS score.

$\mathrm{mRS}$, modified Rankin scale.

postoperative revascularisation is a complex process. Both bypass patency and spontaneous neoangiogenesis from the dual and ischaemic brain tissues contribute to revascularisation in MMD. Thus, we cannott simply exclude the possibility that bypass patency may provide benefits and decrease adverse postoperative events in MMD. Spontaneous neoangiogenesis is rarely observed in other cerebrovascular diseases, such as intracranial aneurysms. The bypass patency is essential to determine the success of the intervention, as well as long-term survival of patients undergoing these procedures (eg, bypass procedures for complex intracranial aneurysms).

The present study has limitations that need to be addressed for an accurate interpretation of our data. First, the major limitation of our study is the retrospective nature of the investigation, and not all patients were followed up on a regular basis. Second, the present study was powered for the surrogate outcome of STA-MCA bypass patency, and not for clinical events. Third, although we performed a propensity score matching to reduce the selection bias between the two groups, the patients' treatment adherence may still impact or bias results. Fourth, the cases at our tertiary referral centre may not be representative of neurosurgical cases nationally. This may render the results prone to potential attrition biases. Fifth, data on the use of other medications apart from aspirin (eg, betablockers, calcium channel blockers) was not consistently collected, which could play a confounding role. Finally, the number of occlusive events in both groups was rather small, and hence, sparse data bias could also be present in the observed results. Further verification using a large prospective cohort is essential.

\section{CONCLUSION}

The overall bypass patency rate in this cohort was $94 \%$. Aspirin is associated with an improved patency rate, and administration may increase the patency rate of bypass grafts in adult patients with ischaemic-onset MMD, without increasing the risk of postoperative haemorrhagic events. Patients with FU bypass patency present a better neurological status than those with FU bypass occlusion. However, our findings are limited by the retrospective nature of this study. Additional studies, especially carefully designed prospective studies, are warranted to address the role of aspirin after bypass procedures.

Contributors JL designed the study, wrote the manuscript, researched the data and contributed to the discussion. GS designed the study, contributed to the discussion and edited the manuscript. YZ, RW, DZ and XC contributed to the discussion and edited the manuscript. HW and JZZ designed the study, researched the data, reviewed/edited the manuscript and contributed to the discussion.

Funding This study is supported by National Key Technology Research and Development Program of the Ministry of Science and Technology of China (2015BAl12B04). Beijing Science and Technology Supporting Plan (D16110000381605). Beijing Municipal Administration of Hospitals' Mission Plan (SML20150501). Beijing Municipal Administration of Hospitals Incubating Program (PX2016034). National Natural Science Foundation of China (81571110,81771234).

Competing interests None declared.

Patient consent for publication Not required.

Ethics approval The study was approved by the IRB of Beijing Tiantan Hospital, Capital Medical University.

Provenance and peer review Not commissioned; externally peer reviewed. Data availability statement The data that support the findings of this study are available from the corresponding author on reasonable request.

Open access This is an open access article distributed in accordance with the Creative Commons Attribution Non Commercial (CC BY-NC 4.0) license, which permits others to distribute, remix, adapt, build upon this work non-commercially, and license their derivative works on different terms, provided the original work is properly cited, appropriate credit is given, any changes made indicated, and the use is non-commercial. See: http://creativecommons.org/licenses/by-nc/4.0/.

ORCID iD

Junlin Lu http://orcid.org/0000-0003-4879-0729

\section{REFERENCES}

1 Kuroda S, Houkin K. Moyamoya disease: current concepts and future perspectives. Lancet Neurol 2008;7:1056-66.

2 Scott RM, Smith ER. Moyamoya disease and moyamoya syndrome. N Engl J Med 2009;360:1226-37.

3 Yoon S, Burkhardt J-K, Lawton MT. Long-term patency in cerebral revascularization surgery: an analysis of a consecutive series of 430 bypasses. J Neurosurg 2018;131:80-7.

4 EC/IC Bypass Study Group. Failure of extracranial-intracranial arterial bypass to reduce the risk of ischemic stroke. Results of an international randomized trial. N Engl J Med 1985;313:1191-200. 
5 Valgimigli M, Bueno H, Byrne RA, et al. 2017 ESC focused update on dual antiplatelet therapy in coronary artery disease developed in collaboration with EACTS. Eur J Cardio Thorac Surg 2018;53:34-78.

6 Lawton MT. Seven bypasses: Tenets and techniques for revascularization. New York: Thieme, 2018: 70.

$7 \mathrm{Lu} \mathrm{J,} \mathrm{Li} \mathrm{Z,} \mathrm{Zhao} \mathrm{Y,} \mathrm{et} \mathrm{al.} \mathrm{Hemorrhagic} \mathrm{transformation} \mathrm{in} \mathrm{ischemic}$ moyamoya disease: clinical characteristics, radiological features, and outcomes. Front Neurol 2020;11:517.

8 , Research Committee on the Pathology and Treatment of Spontaneous Occlusion of the Circle of Willis, Health Labour Sciences Research Grant for Research on Measures for Infractable Diseases. Guidelines for diagnosis and treatment of moyamoya disease (spontaneous occlusion of the circle of Willis). Neurol Med Chir 2012;52:245-66.

9 Suzuki J, Takaku A. Cerebrovascular "moyamoya" disease. Disease showing abnormal net-like vessels in base of brain. Arch Neurol 1969;20:288-99.

$10 \mathrm{Lu} \mathrm{J}$, Zhao Y, Ma L, et al. Predictors and clinical features of transient neurological events after combined bypass revascularization for moyamoya disease. Clin Neurol Neurosurg 2019;186:105505.

11 Li Z, Lu J, Ma L, et al. dl-3-n-butylphthalide for alleviation of neurological deficit after combined extracranial-intracranial revascularization for moyamoya disease: a propensity scorematched analysis. J Neurosurg 2019;132:421-33.
12 Rosenbaum PR, Rubin DB. Constructing a control group using multivariate matched sampling methods that incorporate the propensity score. Am Statian 1985;39:33-8.

13 Liu Z-W, Han C, Zhao F, et al. Collateral circulation in moyamoya disease: a new grading system. Stroke 2019;50:2708-15.

14 Zhao M, Deng X, Zhang D, et al. Risk factors for and outcomes of postoperative complications in adult patients with moyamoya disease. J Neurosurg 2018:80-1.

15 Kraemer M, Berlit P, Diesner F, et al. What is the expert's option on antiplatelet therapy in moyamoya disease? Results of a worldwide survey. Eur J Neurol 2012;19:163-7.

16 Onozuka D, Hagihara A, Nishimura K, et al. Prehospital antiplatelet use and functional status on admission of patients with nonhaemorrhagic moyamoya disease: a nationwide retrospective cohort study (J-ASPECT study). BMJ Open 2016;6:e009942.

17 Zhao Y, Zhang Q, Zhang D, et al. Effect of aspirin in postoperative management of adult ischemic moyamoya disease. World Neurosurg 2017;105:728-31.

$18 \mathrm{Hu} \mathrm{Z}$, Zhang F, Yang Z, et al. Low-dose aspirin promotes endothelial progenitor cell migration and adhesion and prevents senescence. Cell Biol Int 2008;32:761-8.

19 Chen T-G, Chen J-Z, Xie X-D. Effects of aspirin on number, activity and inducible nitric oxide synthase of endothelial progenitor cells from peripheral blood. Acta Pharmacol Sin 2006;27:430-6. 